\title{
Evolution in action: habitat transition from sediment to the pelagial leads to genome streamlining in Methylophilaceae
}

\author{
Michaela M. Salcher (i] ${ }^{1,2} \cdot$ Daniel Schaefle ${ }^{2,3} \cdot{\text { Melissa } \text { Kaspar }^{2} \cdot \text { Stefan M. Neuenschwander }}^{2,4} \cdot$ Rohit Ghai ${ }^{1}$
}

Received: 18 March 2019 / Revised: 14 June 2019 / Accepted: 21 June 2019 / Published online: 10 July 2019

(c) The Author(s) 2019. This article is published with open access

\begin{abstract}
The most abundant aquatic microbes are small in cell and genome size. Genome-streamlining theory predicts gene loss caused by evolutionary selection driven by environmental factors, favouring superior competitors for limiting resources. However, evolutionary histories of such abundant, genome-streamlined microbes remain largely unknown. Here we reconstruct the series of steps in the evolution of some of the most abundant genome-streamlined microbes in freshwaters ("Ca. Methylopumilus") and oceans (marine lineage OM43). A broad genomic spectrum is visible in the family Methylophilaceae (Betaproteobacteria), from sediment microbes with medium-sized genomes (2-3 Mbp genome size), an occasionally blooming pelagic intermediate $(1.7 \mathrm{Mbp})$, and the most reduced pelagic forms $(1.3 \mathrm{Mbp})$. We show that a habitat transition from freshwater sediment to the relatively oligotrophic pelagial was accompanied by progressive gene loss and adaptive gains. Gene loss has mainly affected functions not necessarily required or advantageous in the pelagial or is encoded by redundant pathways. Likewise, we identified genes providing adaptations to oligotrophic conditions that have been transmitted horizontally from pelagic freshwater microbes. Remarkably, the secondary transition from the pelagial of lakes to the oceans required only slight modifications, i.e., adaptations to higher salinity, gained via horizontal gene transfer from indigenous microbes. Our study provides first genomic evidence of genome reduction taking place during habitat transitions. In this regard, the family Methylophilaceae is an exceptional model for tracing the evolutionary history of genome streamlining as such a collection of evolutionarily related microbes from different habitats is rare in the microbial world.
\end{abstract}

\section{Introduction}

Marine and freshwater pelagic habitats are numerically dominated by very small microbes (cell volumes $<0.1 \mu \mathrm{m}^{3}$ ).

Supplementary information The online version of this article (https:// doi.org/10.1038/s41396-019-0471-3) contains supplementary material, which is available to authorized users.

$\square$ Michaela M. Salcher

michaelasalcher@gmail.com

1 Department of Aquatic Microbial Ecology, Institute of Hydrobiology, Biology Centre CAS, Na Sádkách 7, 37005 České Budějovice, Czech Republic

2 Limnological Station, Institute of Plant and Microbial Biology, University of Zurich, Seestrasse 187, 8802 Kilchberg, Switzerland

3 Institute of Medical Microbiology, University of Zurich, Gloriastrasse 28/30, 8006 Zurich, Switzerland

4 Institute for Infectious Diseases, University of Bern, Friedbühlstrasse 51, 3001 Bern, Switzerland
Such microbes seem to be perfectly adapted to nutrient-poor (oligotrophic) conditions by successfully competing for dissolved organic matter and nutrients at low nanomolar concentrations due to higher surface-to-volume ratios and superior transport systems [1]. Small-sized cells also enjoy other benefits such as reduced replication costs and mortality rates by size selective protistan predators [2]. The genomes of such oligotrophs are characterized by being very small (streamlined), with highly conserved core genomes and few pseudogenes, compacted intergenic spacers, reduced numbers of paralogs, and a low genomic GC content $[3,4]$. While genetic drift has been proposed as the evolutionary mechanism behind the reduced genomes of symbionts, parasites and commensals selection driven by environmental factors has been suggested as the primary driving force in the case of free-living oligotrophs [3]. The most abundant organisms on earth, bacteria of the marine SAR11 lineage ("Ca. Pelagibacter ubique", Alphaproteobacteria) serve as models for genome streamlining in the oceans [5] and their freshwater sister lineage LD12 is also 
known to be of similarly small size $[6,7]$. Other examples of aquatic microbes with small cells and reduced genomes can be found among Actinobacteria (marine " $\mathrm{Ca}$. Actinomarina minuta" [8], freshwater " $\mathrm{Ca}$. Nanopelagicales" $[9,10]$, freshwater luna1 lineage $[11,12])$, Thaumarchaeota (marine " $\mathrm{Ca}$. Nitrosopelagicus brevis") [13], and Betaproteobacteria (freshwater " $\mathrm{Ca}$. Methylopumilus planktonicus" [14], marine OM43 lineage [15, 16]).

The latter are methylotrophs that are specialized in using reduced one-carbon $\left(\mathrm{C}_{1}\right)$ compounds like methanol, methylamine, and formaldehyde as sole energy and carbon sources by means of a modular system of different pathways for their oxidation, demethylation, and assimilation [17]. The family Methylophilaceae is among the most important methylotrophs playing a key role in the carbon cycle of aquatic habitats $[17,18]$. Four genera are so far validly described (Methylotenera, Methylobacillus, Methylophilus, and Methylovorus) that mainly inhabit the sediment of freshwater lakes [19-22]. Axenic strains have been also isolated from the pelagial of lakes (" $\mathrm{Ca}$. Methylopumilus") [14] and oceans (lineage OM43) [15, 16, 23]. Freshwater " $\mathrm{Ca}$. Methylopumilus planktonicus" are ubiquitous and abundant in lakes [24] with distinct maxima of up to $11.3 \times 10^{7}$ cells $^{-1}$ (4\% of the total prokaryotic community) during diatom and/or cyanobacterial blooms $[14,25,26]$, indicating that $C_{1}$ substrates supporting their growth are released from primary producers. Members of the coastal marine OM43 lineage display similar temporal patterns with highest numbers $\left(4 \%\right.$ or $8 \times 10^{7}$ cells $\left.1^{-1}\right)$ during phytoplankton blooms [27-29].

In this work, we analysed the evolutionary history of the family Methylophilaceae by comparative genomics. While sediment dwellers have a larger cell and genome size, pelagic lineages are genome streamlined. We hypothesize that the evolutionary origin of the family can be traced back to freshwater sediments, from where these microbes emerged to colonize the plankton of lakes and eventually also crossing the freshwater-marine boundary. The transition from sediments to the pelagial resulted in a pronounced genome reduction and adaptive gene loss has mainly affected functions that are not necessarily required or advantageous in the pelagial or are encoded in redundant pathways. Likewise, genes providing adaptations to oligotrophic conditions might have been transmitted horizontally from indigenous pelagic microbes.

\section{Material and methods}

\section{Isolation of planktonic freshwater methylotrophs}

Novel strains of " $\mathrm{Ca}$. Methylopumilus" and other Methylophilaceae were isolated from the pelagial of Lake Zurich
(CH), Rímov Reservoir (CZ), and Lake Medard (CZ). Dilution to extinction using filtered $(0.2 \mu \mathrm{m})$ and autoclaved water amended with vitamins and amino acids as a medium was used for Lake Zurich [14]. A full-cycle isolation approach [30] was employed for samples from Římov Reservoir and Lake Medard, with filtered water samples $(0.45 \mu \mathrm{m}$ filters $)$, being diluted 1:10 with Artificial Lake Water (ALW) [31] containing vitamins $(0.593 \mu \mathrm{M}$ thiamine, $0.08 \mu \mathrm{M}$ niacin, $0.000074 \mu \mathrm{M}$ cobalamine, $0.005 \mu \mathrm{M}$ paraamino benzoic acid, $0.074 \mu \mathrm{M}$ pyridoxine, $0.081 \mu \mathrm{M}$ pantothenic acid, $0.004 \mu \mathrm{M}$ biotin, $0.004 \mu \mathrm{M}$ folic acid, 0.555

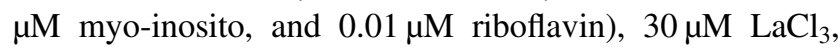
$1 \mathrm{mM}$ methanol, and $0.1 \mathrm{mM}$ methylamine and incubated for 1-2 days at in situ temperatures. This step resulted in a preadaptation of methylotrophs only $\left(\mathrm{C}_{1}\right.$ compounds as sole carbon source) without causing a shift in the assemblage of "Ca. Methylopumilus" as these microbes display slow growth with doubling times of $\sim 2$ days. Thereafter, a dilution-to-extinction technique was employed [14] with approximately one cell per cultivation well in 24-well-plates containing $2 \mathrm{ml}$ of medium. Plates were incubated for 4-6 weeks at in situ temperature and growth in individual wells was checked microscopically and by PCR and Sanger sequencing of $16 \mathrm{~S}$ rRNA genes.

\section{Whole-genome sequencing, assembly, and functional annotation}

Thirty-eight pure cultures of " $\mathrm{Ca}$. Methylopumilus sp." and three Methylophilus sp. were grown in $400 \mathrm{ml} \mathrm{ALW} \mathrm{med-}$ ium supplemented with vitamins, $\mathrm{LaCl}_{3}$, methanol and methylamine for $6-8$ weeks, pelleted by centrifugation, and DNA was isolated with a MagAttract HMW DNA Kit (Qiagen). Thereafter, 550-bp libraries were constructed with the KAPA Hyper Prep Kit (Roche) and paired-end sequences $(2 \times 250 \mathrm{bp})$ were generated on an Illumina MiSeq instrument with a 500-cycle MiSeq Reagent v2 kit (Illumina). Library preparation and sequencing were done at the Genetic Diversity Center Zurich (GDC). Raw reads were quality trimmed with trimmomatic [32], assembled with SPAdes [33] and subsequently mapped to the resulting assemblies with Geneious 9 (www.geneious.com) in order to identify potential assembly errors. Assembly usually resulted in 1-2 large contigs with overlapping ends that mostly could be circularized in silico. In the case of nonoverlapping contigs, genomes were closed by designing specific primers for PCR and Sanger sequencing. Moreover, regions containing low coverage ( $\leq 10$-fold), ambiguities, or anomalies in the mapping were verified by designing specific primers for PCR and Sanger sequencing to produce high-quality reference genomes. Gene prediction was done with PROKKA [34] and annotation was done with an inhouse pipeline [10] based on BLAST searches to NCBI NR, 
COG [35], TIGRFAM [36], and KEGG databases [37]. Metabolic pathways were inferred from KEGG [37] and MetaCyc [38] and manually examined for completeness. Pathways involved in methylotrophy were identified by collecting 1016 reference protein sequences from published genomes of methylotrophs [14, 17, 39-45] and for the sake of completeness, also pathways not common to Methylophilaceae were included (e.g., methane oxidation [46-48], methylovory $[49,50])$. These proteins were classified into 25 modules representing distinct (or sometimes alternative) biochemical transformations relevant to a methylotrophic lifestyle (e.g., M01-methanol oxidation, M02-pyrroloquinoline quinone biosynthesis, etc.; a complete list is provided in Supplementary Table S5). Protein sequences were clustered at $90 \%$ identity and $90 \%$ coverage with cd-hit [51] and the clusters were aligned using muscle [52]. The alignments were converted to HMMs (Hidden Markov Models) using the hmmbuild program in the HMMER3 package [53]. The program hmmsearch was used to scan complete genomes using these HMMs using an $e$-value cutoff of 1e-3. The entire set of HMMs is available as Supplementary Data Set.

\section{Fragment recruitment from metagenomes}

Publicly available metagenomes gained from freshwater sediments $(n=131)$, the pelagial of lakes $(n=345)$, rivers $(n=43)$, estuaries, brackish, and coastal oceanic sites $(n=$ $53)$, as well as open oceans $(n=201)$ were used for fragment recruitment (see Table S2 for sampling sites and SRR accessions). rRNA sequences in genomes were identified with barrnap (http://www.vicbioinformatics.com/software. barrnap.shtml) and masked to avoid biases, and metagenomic reads were queried against the genomes using BLASTN [54] (cutoffs: length $\geq 50 \mathrm{bp}$, identity $\geq 95 \%$, evalue $\leq 1 \mathrm{e}-5$ ). These hits were used to compute RPKG values (number of reads recruited per kb of genome per $\mathrm{Gb}$ of metagenome), which provide a normalized value that is comparable across different genomes and metagenomes.

\section{Comparative genomic analyses}

All publicly available genomes of high quality ( $>95 \%$ completeness, $<20$ scaffolds) affiliated with the family Methylophilaceae (Table S1, $n=37$ ) were downloaded from NCBI and re-annotated for comparative analyses. Average nucleotide identities (ANIs) [55] and average amino acid identities (AAIs) [56] were calculated to discriminate different species and genera. Phylogenomic trees based on conserved concatenated protein sequences (351,312 amino acid sites from 878 proteins for all Methylophilaceae, Fig. 1; 337,501 amino acid sites from 983 proteins for all " $\mathrm{Ca}$. Methylopumilus" spp., Fig. S1) was generated with FastTree [57] (100 bootstraps) after alignment with kalign [58]. Methyloversatilis sp. RAC08 (NZ_CP016448) and " $\mathrm{Ca}$. Methylosemipumilus turicensis" MMS-10A-171 (NZ_LN794158) served as outgroup for the trees displayed in Figs. 1 and S1, respectively. The coreand pan-genome of the family was computed using all-vs.all comparisons of all proteins for each genome using BLASTP ( $\geq 50 \%$ identity and $\geq 50 \%$ coverage cutoffs to define an ortholog). Paralogs in each genome were identified with BLASTP (cutoffs: $\geq 80 \%$ coverage, $\geq 70 \%$ similarity, and $\geq 50 \%$ identity). Closest relatives for proteins putatively transferred horizontally were identified with BLASTP against the NCBI Protein Reference Sequences database (cutoff: $E$ values $\leq 1 \mathrm{e}-5$ ). Trees for individual proteins or concatenated proteins for specific pathways were constructed with RAxML (GAMMA BLOSUM62 model [59]) after alignment with MAFFT v7.388 [60].

\section{Availability of data}

All genomes have been submitted to NCBI under BioProjects PRJNA547471 (BioSamples SAMN11964583-623), PRJEB7927 (BioSample SAMEA3165956), and PRJEB7906 (Biosample SAMEA3165955). The entire set of HMMs related to methylotrophic functions is available as Supplementary Dataset (Dataset 2)

\section{Results and discussion}

\section{Phylogenomics and global occurrence of Methylophilaceae}

Currently, 31 Methylophilaceae genomes of high quality (i.e., $>99 \%$ completeness, $<20$ scaffolds) are publicly available, mostly from axenic isolates from freshwater sediments (Fig. 1, Table S1). We additionally sequenced the genomes of 41 strains of planktonic freshwater strains affiliated with " $\mathrm{Ca}$. Methylopumilus planktonicus" (38 strains) and Methylophilus sp. (3 strains). These microbes were isolated from the pelagial of three different freshwater habitats (Lake Zurich, CH; Rímov Reservoir, CZ; Lake Medard, CZ) by dilution-to-extinction [14, 30]. All novel genomes are of very high quality, i.e., they are complete, with one circular chromosome (Table S1). The 39 strains classified as " $\mathrm{Ca}$. M. planktonicus" by $16 \mathrm{~S}$ rRNA gene sequences analysis (99.94-100\% sequence identity), constitute at least three different species according to average nucleotide and amino acid identity $(<95 \%$ ANI and AAI [61]) (Figs. S1-S4). We tentatively name these three taxa "Ca. Methylopumilus rimovensis" (two strains isolated from Rímov Reservoir), " $\mathrm{Ca}$. Methylopumilus universalis" (29 strains from Lake Zurich and Rímov Reservoir) and the 
a

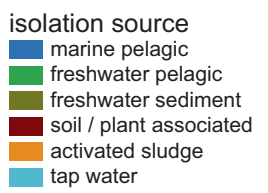

bootstraps $0.99-1$ 0.92

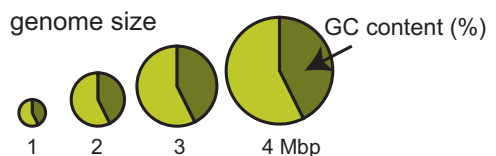

b

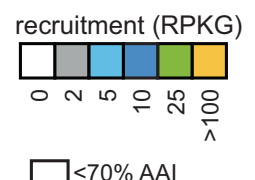

$<70 \%$ AAl

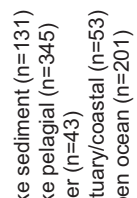

${ }^{*}$ 2-17 scaffolds, genome completeness $>99 \%$

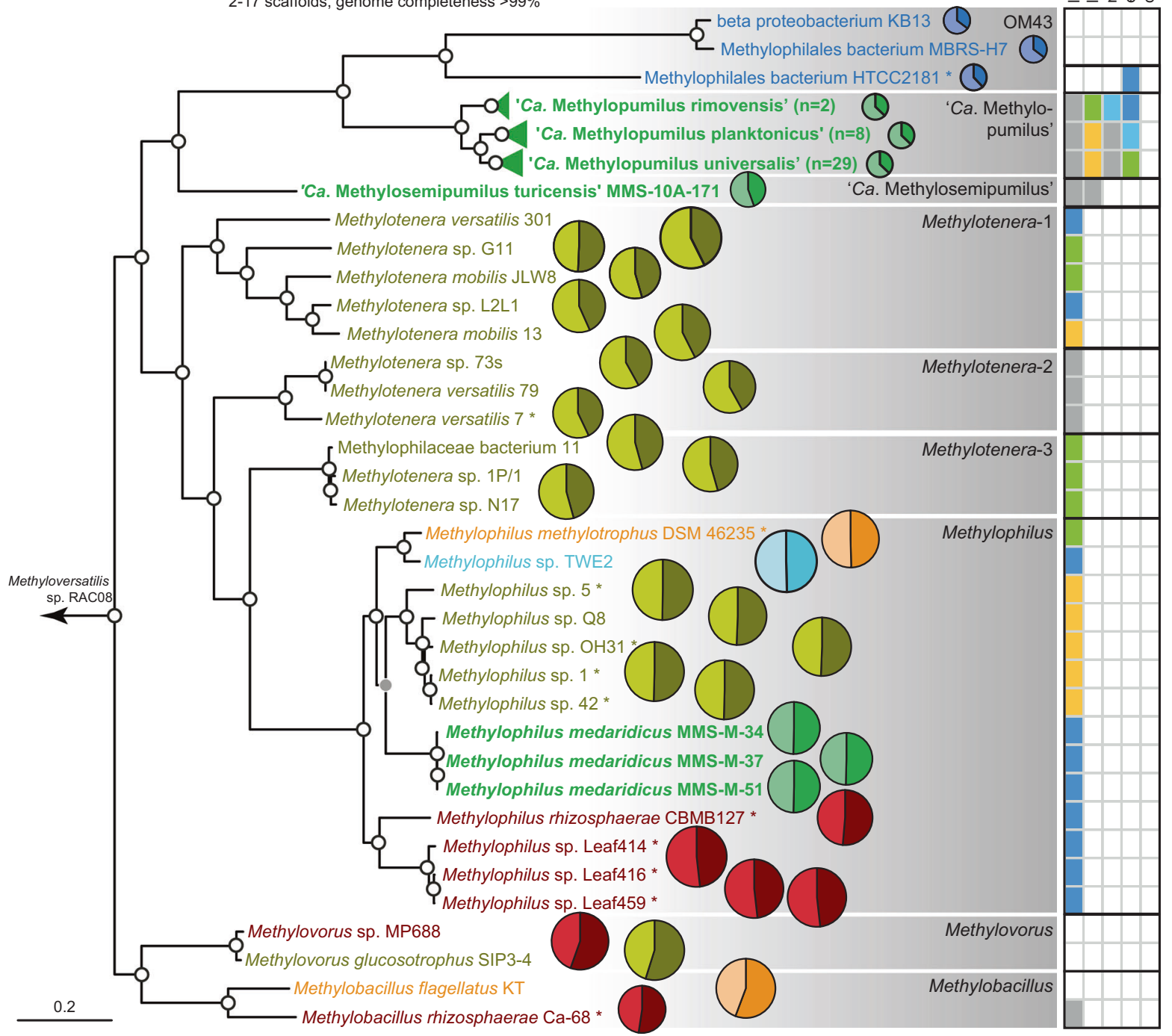

Fig. 1 Phylogeny of Methylophilaceae and their occurrence in different environments. a Phylogenomic tree based on 878 common concatenated proteins (351,312 amino acid sites) with Methyloversatilis sp. RAC08 as outgroup. The 39 complete genomes of " $\mathrm{Ca}$. Methylopumilus sp." are collapsed to the species level, see Fig. S1 for a complete tree. Different genera (70\% AAI cutoff, Fig. S2) are marked by gray boxes. Isolation sources of strains are indicated by different colors and incomplete genomes consisting of $<17$ contigs (estimated completeness $>99 \%$ ) are marked with asterisks. Bootstrap

originally described " $\mathrm{Ca}$. Methylopumilus planktonicus" (8 strains from Lake Zurich; Figs. 1 and S1) [14]. AAI values also suggest that " $\mathrm{Ca}$. M. turicensis" is a different genus (62\% AAI with "Ca. Methylopumilus", Fig. S3) that we tentatively rename to " $\mathrm{Ca}$. Methylosemipumilus turicensis". This reclassification is in line with the recently released Genome Taxonomy Database (GTDB) [62].

values (100 repetitions) are indicated at the nodes, the scale bar at the bottom indicates $20 \%$ sequence divergence. The genome sizes for all strains are shown with circles of proportional size and GC content is depicted within each circle. b Fragment recruitment of public metagenomes from freshwater sediments $(n=131)$, lake pelagial $(n=345)$, rivers $(n=43)$, estuaries and coastal oceans $(n=53)$, and open oceans $(n=201)$. Maximum RPKG values (number of reads recruited per $\mathrm{kb}$ of genome per $\mathrm{Gb}$ of metagenome) for each ecosystem are shown for each genome

Moreover, the genus Methylotenera might be split in different genera and the GTDB suggests a reclassification of several strains to the genus Methylophilus. Our analysis notes a polyphyletic pattern of Methylotenera with three different genera (Methylotenera-1, Methylotenera-2, and Methylotenera-3; Figs. 1a and S3, $>70 \%$ AAI). However, further work is necessary to clarify the formal naming of 
these strains as AAI values are inconclusive and the proposed hard cutoff of $65 \%$ AAI for genus delineation is not met for most members of Methylophilaceae. Three novel pelagic Methylophilus sp. isolates (MMS-M-34, MMS-M37, and MMS-M-51) constitute a novel species that we tentatively named $M$. medardicus, with closest hits to isolates from freshwater sediment. These strains might originate from the same clone, as they were gained from the same sample from Lake Medard and were 100\% identical in their genome sequence. M. mediardicus seem to be not abundant in the pelagial of lakes, as indicated by recruitments from 345 different pelagic freshwater metagenomic datasets, however they could be readily detected in relatively high proportions in sediment metagenomes (Fig. 1b, Table S2). Sediments also appear to be the main habitat of other Methylophilus and Methylotenera. The three strains isolated from marine systems, that were referred to as OM43 lineage [15, 16, 23], form two different genera based on AAI (Fig. S3). However, none appear to be abundant in the open ocean (Fig. 1b), and only strain HTCC2181 could be detected in estuarine/coastal metagenomes, although lineage OM43 has been repeatedly reported in coastal oceans by CARD-FISH, where they can reach up to $4 \%$ or $0.8 \times 10^{5}$ cells ml $^{-1}$ during phytoplankton blooms [28, 29]. It is thus likely that other, more abundant strains of OM43 still await isolation. "Ca. Methylopumilus spp." on the other hand, were found in moderate proportions in estuarine/coastal systems, but their main habitat is clearly the pelagial of lakes, where they are highly abundant (Fig. 1b), as previously reported based on CARD-FISH analyses [14, 63], 16S rRNA gene amplicon sequencing [24, 64-66], and metagenomics [67, 68]. All " $\mathrm{Ca}$. Methylopumilus", especially " $\mathrm{Ca}$. M. rimovensis" were also prevalent in rivers (Fig. 1b).

\section{Genome streamlining in pelagic strains}

The genomes of pelagic freshwater " $\mathrm{Ca}$. Methylopumilus sp." $(n=39)$ and the marine OM43 lineage $(n=3)$ are characterized by very small sizes $(1.26-1.36 \mathrm{Mbp})$ and a low genomic GC content (35.3-37.7\%) (Table S1, Figs. 2 and S5). "Ca. Methylosemipumilus turicensis" MMS-10A171 has a slightly larger genome $(1.75 \mathrm{Mbp})$ with higher GC content (44.5\%), while all other Methylophilaceae have genome sizes $>2.37 \mathrm{Mbp}(\max .3 .25 \mathrm{Mbp})$ and a higher GC content (41.9-55.7\%, average 47.3\%). A highly significant relationship between genome size and GC content, length of intergenic spacers, coding density, mean CDS length, number of overlapping CDS, paralogs, and numbers of genes involved in sensing of the environment (i.e., histidine kinases and sigma factors) was evident (Fig. 2). All these features have been proposed to be relevant for genome streamlining [3] with freshwater " $\mathrm{Ca}$. Methylopumilus" and marine OM43 displaying the most reduced forms and " $\mathrm{Ca}$. Methylosemipumilus turicensis" presenting an intermediate state (Table S1). Moreover, we observed a negative relationship between genomic GC content and stop-codon usage of TAA instead of TAG, as well as a preferred amino acid usage of lysine instead of arginine (Fig. 2), both suggested to be involved in nitrogen limitation [4]. Furthermore, amino acids with less nitrogen and sulfur and more carbon atoms were favorably encoded by the genomestreamlined microbes (Figs. 2, S5 and S6).

\section{Adaptive gene loss during habitat transition from the sediment to the pelagial}

The core genome of the family Methylophilaceae consists of 664 protein families (4.3\% of the pangenome) and an open pangenome of $>15,000$ protein families, while the streamlined genomes of " $\mathrm{Ca}$. Methylopumilus" have a highly conserved core (48\%, Fig. S7). By contrast, sediment Methylophilaceae have a larger pangenome with a more modular assortment featuring several redundant pathways for methylotrophic functions [18] and a large fraction of proteins overlapping with " $\mathrm{Ca}$. M. turicensis", indicating a high evolutionary relatedness (Fig. S7). It appears that both extant pelagic and sediment Methylophilaceae shared a common sediment-dwelling methylotrophic ancestor. While one lineage (Methylotenera and Methylophilus) retains the ancestral character (large genomes) of the common ancestor, the other lineage diversified towards a pelagic lifestyle (“Ca. M. turicensis", "Ca. Methylopumilus", and OM43). Remarkably, "Ca. M. turicensis" appears to constitute an early diverging lineage that displays somewhat mixed characteristics of both sediment and truly pelagic forms ("Ca. Methylopumilus" and OM43), not only in its phylogenetic position, but also in genomic characteristics. Monitoring data from Lake Zurich showed consistently high cell densities of " $\mathrm{Ca}$. Methylopumilus", while " $\mathrm{Ca}$. M. turicensis" was mostly below detection limits except for a 3month phase in 1 year where they reached high numbers in the hypolimnion [14]. Moreover, also fragment recruitment from freshwater metagenomes showed a global occurrence of " $C a$. Methylopumilus" in very high relative proportions, while "Ca. M. turicensis" was less prevalent (Fig. 1b). This hints again at the somewhat transitional character of " $\mathrm{Ca}$. M. turicensis" (occasional "bloomer") that is not as perfectly adapted to the pelagial as " $\mathrm{Ca}$. Methylopumilus".

We tested the hypothesis of adaptive gene loss driven by evolutionary selection during the transition from sediment to the pelagial by comparative genomics of metabolic modules of Methylophilus, Methylotenera, "Ca. M. turicensis", "Ca. Methylopumilus", and marine OM43 strains. Methylobacillus and Methylovorus were excluded as they seem too distantly related and also not very abundant in lake 

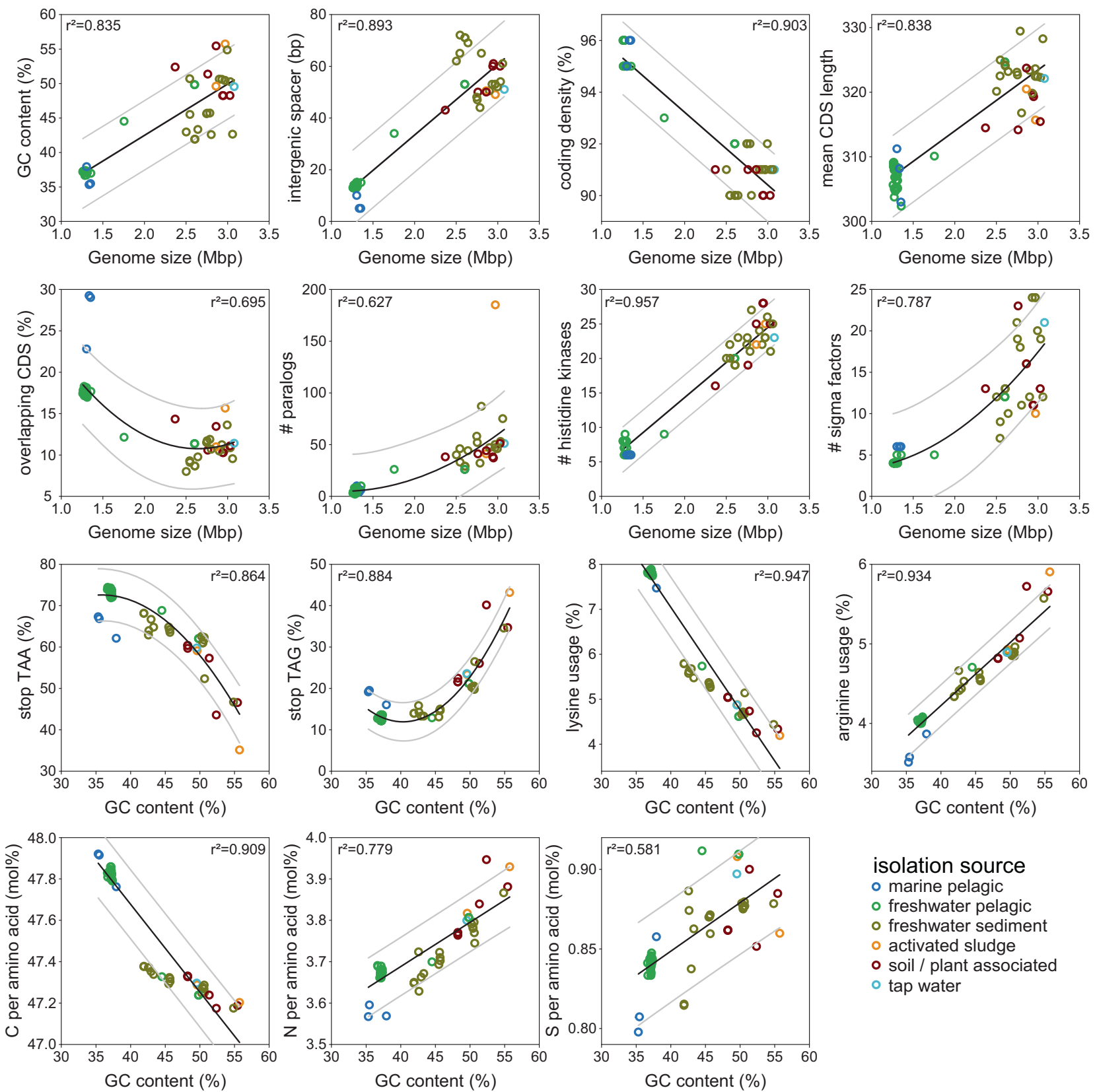

\section{isolation source}

- marine pelagic

- freshwater pelagic

- freshwater sediment

- activated sludge

- soil / plant associated

- tap water

Fig. 2 Genome streamlining in Methylophilaceae. Significant relationships between genome sizes (Mbp) and genomic GC content (\%), lengths of intergenic spacers (bp), coding density (\%), mean CDS length (bp), overlapping CDS (\%), number of paralogs, histidine

sediments (Fig. 1b, Table S2). The most pronounced differences in the genetic make-up of sediment vs. pelagic strains were detected in motility and chemotaxis (Figs. 3 and 4), with all Methylophilus and all but two Methylotenera strains having flagella and type IV pili, while the planktonic strains have lost mobility and also greatly reduced the number of two-component regulatory systems and sigma factors. A large number of membrane transporters for inorganic compounds was detected exclusively in sediment Methylophilaceae, while this number is reduced in kinases, and sigma factors, and significant relationships between genomic GC content (\%) and TAA stop codon, TAG stop codon, lysine, and arginine usage $(\%)$ and $\mathrm{C}, \mathrm{N}$, and $\mathrm{S}$ atoms per amino acid $(\mathrm{mol} \%)$

"Ca. M. turicensis" and even more in "Ca. Methylopumilus" and OM43 (Fig. 4, Table S3). Moreover, Methylophilus and Methylotenera encode multiple pathways for nitrogen acquisition, with transporters for ammonia, nitrate, nitrite, taurine, cyanate, or urea, and pathways for urea or cyanate utilization (Fig. 3, Table S4) [69]. "Ca. M. turicensis", on the other hand, has only transporters for nitrate/taurine and ammonia (Amt family), and "Ca. Methylopumilus" and marine OM43 only carry ammonia transporters. All sediment Methylophilaceae further possess genes for 


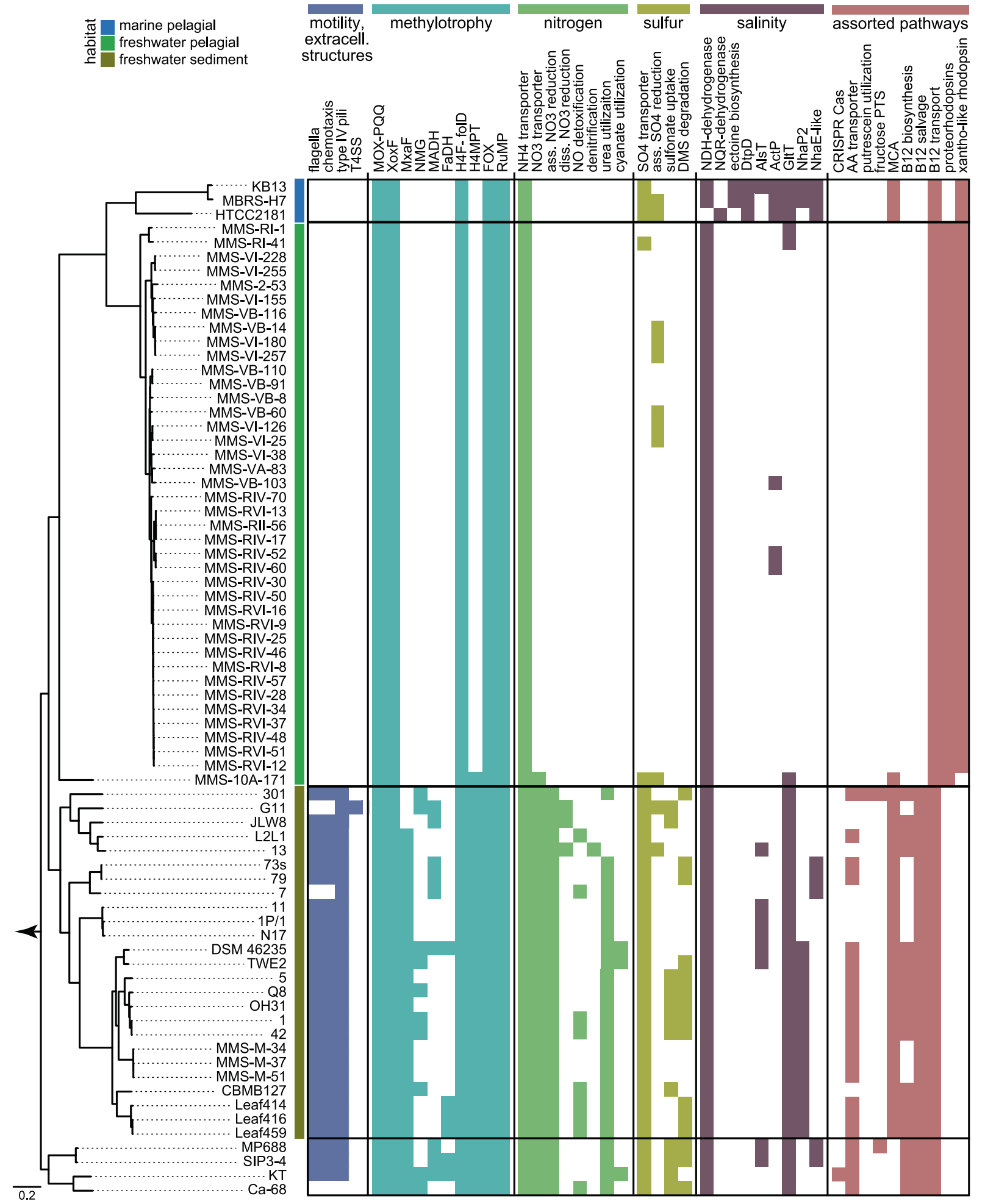

assimilatory nitrate reduction to ammonia, some for dissimilatory nitrate reduction to nitrous oxide or detoxification of nitric oxide (quinol type of norB) [70] and Methylotenera mobilis 13 is a complete denitrifier $[69,71,72]$, while none of the pelagic strains have any genes involved in nitrate reduction (Figs. 3 and 4, Table S4). Ammonia is the main microbial nitrogen source in the epilimnion of lakes and oceans, while nitrate and other compounds like urea, taurine, or cyanate are more abundant in deeper, oxygenated layers and the sediment 
Fig. 3 Metabolic modules in Methylophilaceae. Presence of selected metabolic modules in Methylophilaceae strains. For details on phylogenomic tree see Figs. 1 and S1, for details on pathways see Table S4. T4SS: type 4 secretion system; MOX-PQQ: methanol oxidation and pyrroloquinoline quinone biosynthesis; XoxF/MxaF: methanol dehydrogenase XoxF/MxaF; NMG: N-methyloglutamate pathway; MADH: methylamine dehydrogenase; $\mathrm{FaDH}$ : formaldehyde dehydrogenase; H4F-folD: $\mathrm{H}_{4}$-linked formaldehyde oxidation, folD form; H4MPT: $\mathrm{H}_{4}$ MPT-linked formaldehyde oxidation; FOX: formate oxidation; RuMP: ribulose monophosphate cycle; ass. NO3 reduction: assimilatory nitrate reduction; diss. NO3 reduction: dissimilatory nitrate reduction; ass. SO4 reduction: assimilatory sulfate reduction; $\mathrm{NDH}$-dehydrogenase: $\mathrm{H}^{+}$-translocating $\mathrm{NADH}$ :quinone oxidoreductase; NQR-dehydrogenase: $\mathrm{Na}^{+}$-translocating NADH:quinone oxidoreductase; DtpD: dipeptide/tripeptide permease DtpD; AlsT: sodium:alanine symporter AlsT; ActP: sodium:acetate symporter ActP; GltT: sodium:dicarboxylate symporter GltT; NhaP2: sodium: proton antiporter NhaP2; NhaE-like: sodium:proton antiporter NhaE; AA transporter: amino acid transporters; fructose PTS: fructosespecific phosphotransferase system; MCA: methylcitric acid cycle

$[14,73,74]$. Therefore, an adaptation to ammonium uptake might be advantageous for pelagic microbes.

Furthermore, a high diversity of pathways involved in sulfur metabolism was detected in Methylophilaceae, with the genome-streamlined strains representing the most reduced forms again. All Methylophilus, Methylotenera, "Ca. Methylosemipumilus turicensis", and one strain of "Ca. Methylopumilus rimovensis" encode ABC transporters for sulfate uptake, and a sulfate permease was annotated in OM43 and several sediment Methylophilaceae, while the majority of " $\mathrm{Ca}$. Methylopumilus" lack these transporters (Figs. 3 and 4). Canonical assimilatory sulfate reduction seems to be incomplete in most Methylophilaceae, as adenylyl sulfate kinase cys $C$ was annotated only in a few strains (Table S4). Thus, the mode of sulfite generation remains unclear, with unknown APS kinases or other links from APS to sulfite. Methylophilus rhizosphaera encodes genes for dissimilatory sulfate reduction and most sediment strains possess $\mathrm{ABC}$ transporters for alkanesulfonates, most likely transporting methylsulfonate that can be oxidized to sulfite by methanesulfonate monooxygenases generating formaldehyde as by-product. Dimethyl sulfide seems to be a source for sulfur and formaldehyde, as well as dimethylsulfoxide and dimethyl monooxygenases are present in several sediment Methylophilaceae, but absent in all pelagic strains. It is thus still unclear how " $\mathrm{Ca}$. Methylopumilus" fuel their sulfur demand, especially as they grow in a defined medium containing sulfate $\left(200 \mu \mathrm{M} \mathrm{MgSO}_{4} ; 160\right.$ $\mu \mathrm{M} \mathrm{CaSO}{ }_{4}$ ) and vitamins as sole sulfur sources.

All Methylophilaceae have complete pathways for the biosynthesis of amino acids and vitamins, with the exception of cobalamin (vitamin B12) that was lacking in the pelagic lineages (" $\mathrm{Ca}$. M. turicensis", "Ca . Methylopumilus", and marine OM43), while either the complete biosynthesis or the salvage pathway was present in the sediment isolates (Figs. 3 and 4, Table S4). However, putative cobalamin transporters were annotated in all isolates.

The methylcitric acid (MCA) pathway for oxidizing propionate via methylcitrate to pyruvate is present in Methylotenera, Methylophilus, "Ca. M. turicensis", and the marine OM43, but absent in all " $\mathrm{Ca}$. Methylopumilus" strains, suggesting it has been selectively lost in these organisms. All genes were arranged in a highly conserved fashion, with the exception of " $\mathrm{Ca}$. M. turicensis" having a bifunctional aconitate hydratase 2/2-methylisocitrate dehydratase ( $p r p D / a c n B)$ gene and $a c n B$ genes being located in different genomic regions in OM43 and " $\mathrm{Ca}$. M. turicensis" (possessing two copies), however, with high synteny of flanking genes (Fig. S8). Phylogenetic analysis of the MCA gene cluster resulted in genus-specific branching, and notably, the MCA pathway of OM43 is most closely related to that of "Ca. M. turicensis" (Fig. S8), suggesting it was retained in the OM43 lineage after divergence from a common ancestor of OM43 and " $\mathrm{Ca}$. M. turicensis".

\section{Genome streamlining leading to a loss of redundant methylotrophic pathways}

Some of the sediment dwellers seem to be facultative methylotrophs, as $\mathrm{ABC}$ transporters for amino acids were annotated (Figs. 3 and 4, Table S3). Methylotenera versatilis 301 additionally encodes a fructose-specific phosphotransferase system (PTS) and a 1-phosphofructokinase, as well as transporters for putrescine uptake and the subsequent pathway for its degradation. "Ca. M. turicensis" might also be a facultative methylotroph, as it possesses a PTS system for cellobiose, while this (as well as amino acid transporters) is lacking in all " $\mathrm{Ca}$. Methylopumilus" and OM43 strains, making them obligate methylotrophs. These observations suggest that the ancestor of both pelagic and sediment lineages was also a facultative methylotroph and that obligate methylotrophy emerged only in the truly pelagic strains.

Remarkably, also pathways involved in methylotrophy were reduced in the course of genome streamlining with the sediment-dwelling Methylophilus and Methylotenera having the most complete modules for $\mathrm{C}_{1}$ compound oxidation, demethylation, and assimilation (Figs. 3 and 4, Table S4). They also encode multiple types of methanol dehydrogenases (up to five different types in single strains), while the pelagic forms possess only XoxF4-1 (Fig. S9). Moreover, the latter encode neither traditional methylamine-dehydrogenases nor the N-methylglutamate pathway for methylamine oxidation. Thus, the mode of methylamine uptake is still unclear, although it has been experimentally demonstrated that some pelagic strains can utilize this $\mathrm{C}_{1}$ substrate $[14,75]$. However, also nearly half of the sediment strains lack these well-described pathways 
a Sediment Methylophilaceae (Methylotenera + Methylophilus) vs. 'Ca. Methylosemipumilus turicensis' (Mtur)

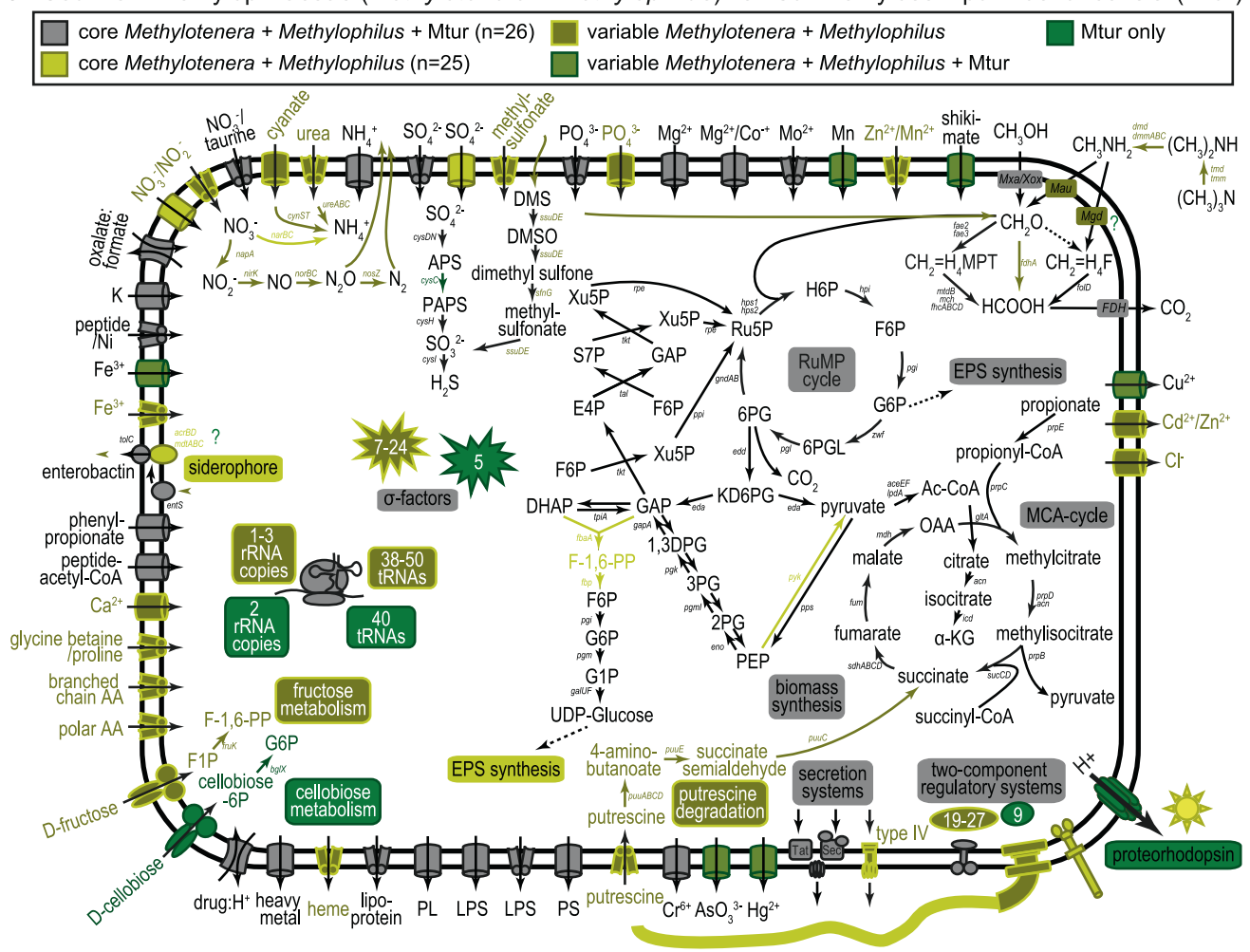

b 'Ca. Methylosemipumilus turicensis' (Mtur) vs. 'Ca. Methylopumilus' (Mpum) vs. marine OM43

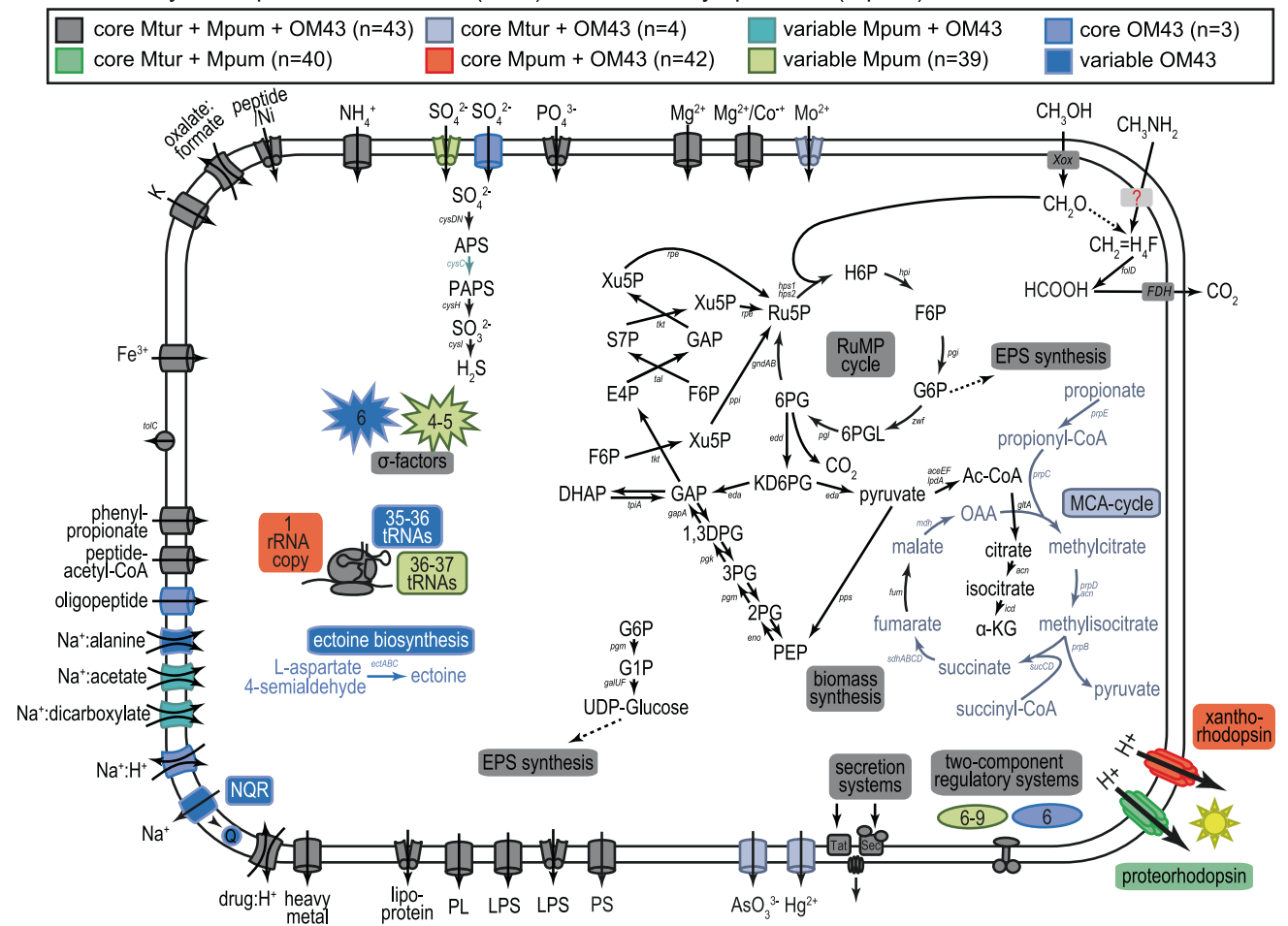

Fig. 4 Comparative metabolic maps of different taxa of Methylophilaceae. a Comparison of the core metabolism in sediment Methylophilaceae (Methylotenera and Methylophilus) vs. "Ca. Methylosemipumilus turicensis" (Mtur). b Comparison of the core metabolism in "Ca. Methylosemipumilus turicensis" (Mtur) vs. "Ca. Methylopumilus" (Mpum) vs. marine OM43. For details on pathways see Table S4 
in a patchy manner only partly reflected by phylogeny, therefore it is likely that methylamine utilization is not a common feature within Methylophilaceae, or that alternative routes of its oxidation still await discovery [69]. Formaldehyde oxidation can be achieved via three alternative routes, and only four Methylophilus strains encode all of them, i.e., all others lack a formaldehyde dehydrogenase. All Methylophilus and Methylotenera as well as "Ca. M. turicensis" carry genes for the tetrahydromethanopterin $\left(\mathrm{H}_{4} \mathrm{MPT}\right)$ pathway, but none of the " $\mathrm{Ca}$. Methylopumilus" and OM43 strains. Therefore, the only route for formaldehyde oxidation in these genome-streamlined microbes is the tetrahydrofolate $\left(\mathrm{H}_{4} \mathrm{~F}\right)$ pathway, which includes the spontaneous reaction of formaldehyde to $\mathrm{H}_{4} \mathrm{~F}$ and is thought to be relatively slow $[14,17]$. The ribulose monophosphate (RuMP) cycle for formaldehyde assimilation/oxidation and formate oxidation via formate dehydrogenases was annotated in all Methylophilaceae, while none of them possess other potential methylotrophic modules such as the serine cycle, the ethylmalonyl-CoA-pathway for glyoxylate regeneration, a glyoxylate shunt, nor the Calvin-Benson-Bassham cycle for $\mathrm{CO}_{2}$ assimilation, as already previously noted to be lacking in Methylophilaceae [17]. Thus, the core methylotrophic modules in Methylophilaceae contain methanol oxidation via XoxF methanol dehydrogenases, formaldehyde oxidation via the $\mathrm{H}_{4} \mathrm{~F}$ pathway, the RuMP cycle, and formate oxidation (Fig. 3, Table S4) $[17,69,76]$. The majority of genes encoding these pathways were organized in operon structures or

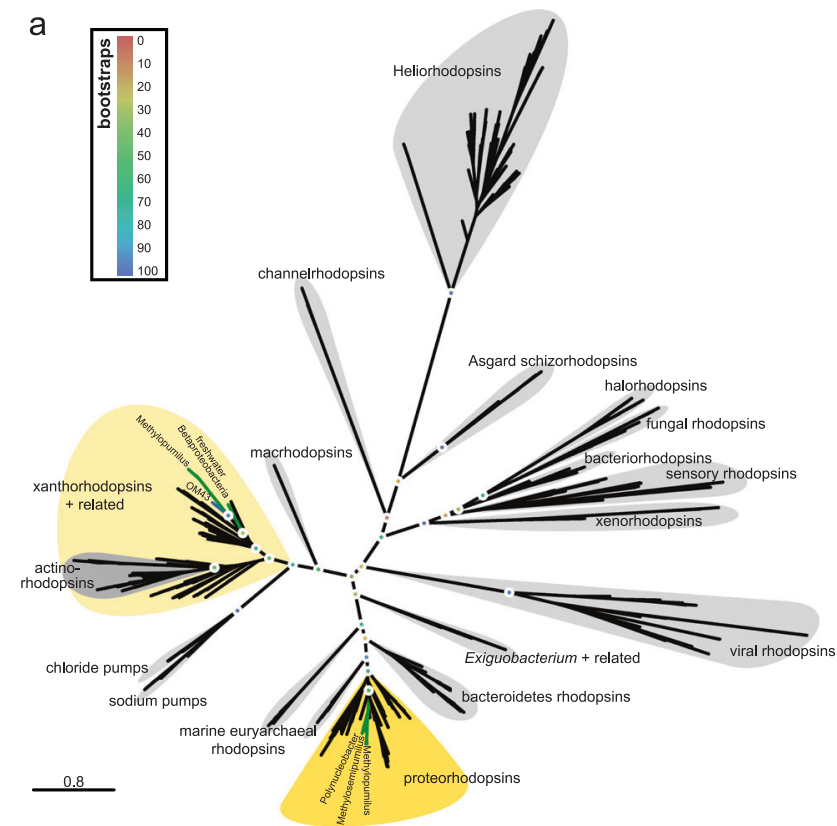

Fig. 5 Horizontal gene transfers of two different rhodopsins. a Phylogenetic tree (RAxML, 100 bootstraps) of different rhodopsin types. See Fig. S12 for details of closely related proteo- and xantho-like rhodopsins of Methylophilaceae. b Arrangement and protein similarity found in close vicinity to each other with high synteny and phylogenetically reflecting the overall phylogeny of the family (Figs. S10 and S11).

\section{Photoheterotrophy as adaptation to oligotrophic pelagic conditions}

Rhodopsins are light-driven proton pumps producing ATP that fuel e.g., membrane transporters [77] and play important roles during carbon starvation [78] in oligotrophic aquatic environments. Therefore, the acquisition of rhodopsins is proposed to be powerful adaptations to the pelagial. "Ca. Methylosemipumilus turicensis" seems to have acquired a proteorhodopsin and the complete pathway for retinal biosynthesis via horizontal gene transfer (HGT) from the abundant freshwater microbe Polynucleobacter cosmopolitanus $(81 \%$ amino acid similarity, Figs. 5 and S12a). Interestingly, " $\mathrm{Ca}$. Methylopumilus spp." carry, in addition to a proteorhodopsin highly similar to " $\mathrm{Ca}$. M. turicensis" (78.3-79.5\% similarity), a second rhodopsin gene inserted between the proteorhodopsin and the retinal biosynthesis cluster (Fig. 5). This xantho-like rhodopsin was most likely gained from rare freshwater Betaproteobacteria (Janthinobacterium lividum, Massilia psychrophila; 49.4-53.5\% similarity, Fig. S12b), however, the binding of a carotenoid antenna seems unlikely due to the replacement of a glycine with tryptophan in position 156 , suggesting it also functions as a proteorhodopsin (Fig. S12c) [79, 80]. Both rhodopsins are tuned to green

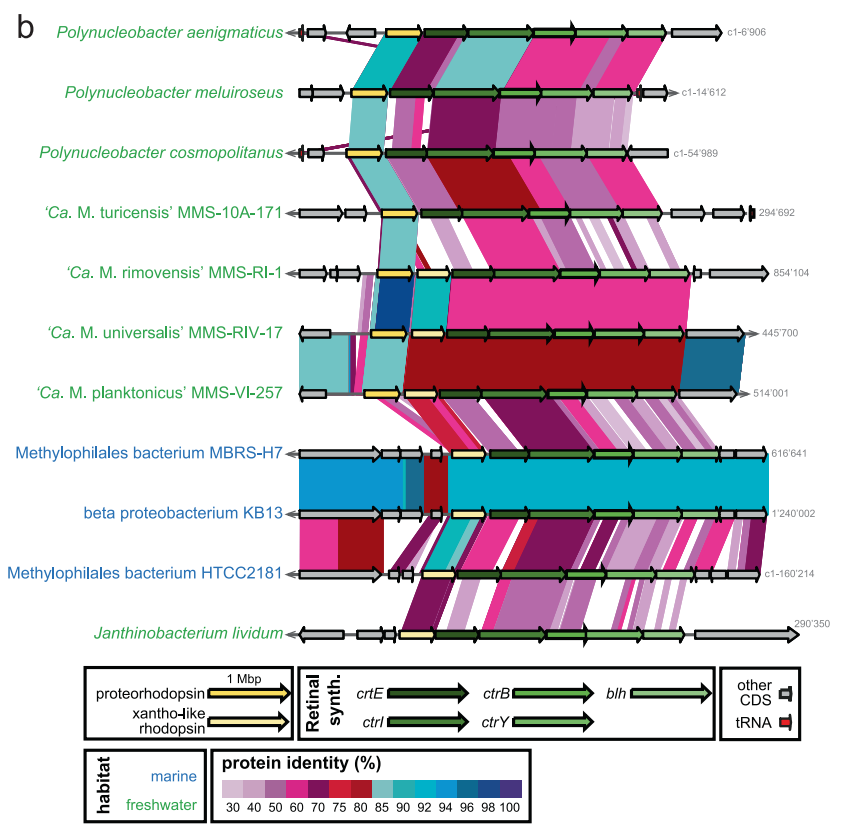

of rhodopsins and the retinal biosynthesis gene cluster in " $\mathrm{Ca}$. Methylopumilus spp.", " $\mathrm{Ca}$. Methylosemipumilus turicensis", marine OM43, and other freshwater microbes with closely related rhodopsin types 
light, which is common in freshwaters [81] and possess the canonical DxxxK retinal binding motif in helix-7 that is characteristic of proton pumping rhodopsins [82]. The marine OM43 lineage only carry the xantho-like rhodopsin (59.0-63.9\% similarity to " $\mathrm{Ca}$. Methylopumilus"). It is unclear if the proteorhodopsin was never present in the marine lineage or was lost subsequently, and if so, the reasons for a secondary loss remain enigmatic as two rhodopsins would provide an even better adaptation to oligotrophic waters than one.

\section{The second transition from freshwater pelagial to the marine realm is characterized by adaptations to a salty environment}

The second habitat transition across the freshwater-marine boundary does not appear to involve genome streamlining, as genomes of pelagic freshwater and marine methylotrophs are of similar small size and low GC content (Figs. 1 and 2). We hypothesize that this transition had less impact on the lifestyle (purely planktonic, oligotrophic) but required specific adaptations to the marine realm that were mainly acquired by HGT, and as suggested by the long branches in the phylogenetic tree (Fig. 1), multiple, rapid changes in existing genes. Besides the MCA pathway and the proteorhodopsin, no major rearrangements or reductions in general metabolic pathways were detected in marine OM43 in comparison with freshwater " $\mathrm{Ca}$. Methylopumilus". However, several adaptations to higher salt concentrations could be identified (Figs. 3 and 4). Salinity is one of the most important obstacle in freshwater-marine colonization, and successful transitions have occurred rarely during the evolution of Proteobacteria [83, 84]. Main adaptations to higher salinities involve genes for osmoregulation and inorganic ion metabolism that might have been acquired from the indigenous community by HGT. For example, genes regulating the $\mathrm{Na}^{+}$-dependent respiratory chain $\left(\mathrm{Na}^{+}\right.$-translocating NADH:quinone oxidoreductase, NQR, Fig. S13) have been transmitted from the marine Roseobacter lineage to strain HTCC2181, a member of the OM43 lineage [16, 84]. The NQR system provides energy by generating a sodium motif force, yet, the sodium pumping might also be an adaptation to enhanced salinities [85]. All other Methylophilaceae possess the energetically more efficient $\mathrm{H}^{+}$-translocating type (NDH), which works better under low salinity conditions and is thus common in freshwater microbes [85].

Ectoine, a compatible solute along with glycine betaine, helps organisms survive extreme osmotic stress by acting as an osmolyte [86]. Ectoine is synthesized from L-aspartate 4semialdehyde, the central intermediate in the synthesis of amino acids of the aspartate family. Two marine OM43 strains (KB13 and MBRS-H7) encode this pathway followed by sodium:proline symporter putP arranged in high synteny and protein similarity with marine/hypersaline sediment microbes, thus it is likely that both components were gained via HGT (Fig. S14). A second copy of the putP symporter was common to all Methylophilaceae (data not shown). Also a dipeptide/tripeptide permease unique for the marine OM43 lineage seems to be transferred horizontally, either from marine Bacteroidetes or sediment-dwelling Sulfurifustis (Gammaproteobacteria, Fig. S15). Other putative membrane compounds involved in sodium transport in marine OM43 include a sodium:alanine symporter (AlsT, Fig. S16a), a sodium:acetate symporter (ActP, Fig. S16b), a sodium:dicarboxylate symporter (GltT, Fig. S16c), a sodium:proton antiporter (NhaP, Fig. S16d), and another putative sodium:proton antiporter (NhaE-like, Fig. S16e). Although also several other Methylophilaceae carry some of these sodium transporters, they are only distantly related to OM43, thus they might be acquired horizontally. Conversely, ActP and GltT of OM43 are most closely related to three " $\mathrm{Ca}$. M. universalis" strains and the two " $\mathrm{Ca}$. M. rimovensis" strains, respectively (Fig. S16b, c). Both symporters are related to microbes from freshwater and marine habitats, hinting to some yet unknown lineages related to both OM43 and " $\mathrm{Ca}$. Methylopumilus" most likely thriving in the freshwater-marine transition zone.

\section{Conclusions}

Our study provides first genomic evidence that the ancestors of genome-streamlined pelagic Methylophilaceae can be traced back to sediments with two habitat transitions occurring in the evolutionary history of the family. The first from sediments to the pelagial is characterized by pronounced genome reduction driven by selection pressure for relatively more oligotrophic environmental conditions. This adaptive gene loss has mainly affected functions that (i) are not necessarily required in the pelagial (e.g., motility, chemotaxis), (ii) are not advantageous for survival in an oligotrophic habitat (e.g., low substrate affinity transporters), and (iii) are encoded in redundant pathways (e.g., formaldehyde oxidation). Likewise, (iv) genes providing adaptations to oligotrophic conditions have been transmitted horizontally from indigenous pelagic microbes (e.g., rhodopsins). "Ca. M. turicensis" was identified as transitional taxon, retaining multiple ancestral characters while also gaining adaptations to the pelagial. The second habitat transition across the freshwater-marine boundary did not result in further genome streamlining, but is characterized by adaptations to higher salinities acquired by HGT. In this regard, the family Methylophilaceae is an exceptional model for tracing the evolutionary history of genome streamlining as such a collection of evolutionarily related 
microbes from different habitats is practically unknown for other similarly abundant genome-streamlined microbes (e.g., "Ca. Pelagibacterales", "Ca. Nanopelagicales”).

Acknowledgements We thank the team of the Genetic Diversity Center Zurich (GDC) for providing sequencing facilities and help with library preparation. Thomas Posch and Eugen Loher are acknowledged for help in sampling of Lake Zurich, Petr Znachor, Pavel Rychtecký, and Jiří Nedoma for help in sampling of Řimov Reservoir and Lake Medard. We thank four anonymous reviewers for helpful and supportive comments on an earlier version of this manuscript. MMS was supported by the research grants 19-23469S (Grant Agency of the Czech Republic) and 310030_185108 (Swiss National Science Foundation). RG was supported by the research grant 17-04828S (Grant Agency of the Czech Republic). Sampling for the isolation of novel strains from Lake Zurich was supported by the SNF D-A-CH project 310030E-160603/1 awarded to Thomas Posch.

Author contributions MMS conceived the project, isolated and sequenced the strains, and analyzed the data and wrote the paper. DS, MK, and SMN sequenced the strains and contributed to data analyses. RG developed programs for analysis and contributed to data analyses. All authors helped to interpret the results and contributed to writing the paper.

\section{Compliance with ethical standards}

Conflict of interest The authors declare that they have no competing interests.

Publisher's note: Springer Nature remains neutral with regard to jurisdictional claims in published maps and institutional affiliations.

Open Access This article is licensed under a Creative Commons Attribution 4.0 International License, which permits use, sharing, adaptation, distribution and reproduction in any medium or format, as long as you give appropriate credit to the original author(s) and the source, provide a link to the Creative Commons license, and indicate if changes were made. The images or other third party material in this article are included in the article's Creative Commons license, unless indicated otherwise in a credit line to the material. If material is not included in the article's Creative Commons license and your intended use is not permitted by statutory regulation or exceeds the permitted use, you will need to obtain permission directly from the copyright holder. To view a copy of this license, visit http://creativecommons. org/licenses/by/4.0/.

\section{References}

1. Button DK. Biochemical basis for whole-cell uptake kinetics: specific affinity, oligotrophic capacity, and the meaning of the Michaelis constant. Appl Environ Microbiol. 1991;57:2033-8.

2. Pernthaler J. Predation on prokaryotes in the water column and its ecological implications. Nat Rev Microbiol. 2005;3:537-46.

3. Giovannoni SJ, Cameron Thrash J, Temperton B. Implications of streamlining theory for microbial ecology. ISME J. 2014;8:1553-65.

4. Luo H, Thompson LR, Stingl U, Hughes AL. Selection maintains low genomic GC content in marine SAR11 lineages. Mol Biol Evol. 2015;32:2738-48

5. Giovannoni SJ, Tripp HJ, Givan S, Podar M, Vergin KL, Baptista $\mathrm{D}$, et al. Genome streamlining in a cosmopolitan oceanic bacterium. Science. 2005;309:1242-5.
6. Salcher MM, Pernthaler J, Posch T. Seasonal bloom dynamics and ecophysiology of the freshwater sister clade of SAR11 bacteria "that rule the waves" (LD12). ISME J. 2011;5:1242-52.

7. Henson MW, Lanclos VC, Faircloth BC, Thrash JC. Cultivation and genomics of the first freshwater SAR11 (LD12) isolate. ISME J. 2018;12:1846-60.

8. Ghai R, Mizuno CM, Picazo A, Camacho A, Rodriguez-Valera F. Metagenomics uncovers a new group of low GC and ultra-small marine Actinobacteria. Sci Rep. 2013;3:2471.

9. Kang I, Kim S, Islam MR, Cho J-C. The first complete genome sequences of the acI lineage, the most abundant freshwater Actinobacteria, obtained by whole-genome-amplification of dilutionto-extinction cultures. Sci Rep 2017;7:42252.

10. Neuenschwander SM, Ghai R, Pernthaler J, Salcher MM. Microdiversification in genome-streamlined ubiquitous freshwater Actinobacteria. ISME J. 2018;12:185.

11. Hahn MW, Schmidt J, Taipale SJ, Doolittle WF, Koll U. Rhodoluna lacicola gen. nov., sp. nov., a planktonic freshwater bacterium with stream-lined genome. Int J Syst Evol Microbiol. 2014;64:3254-63.

12. Kang I, Lee K, Yang S-J, Choi A, Kang D, Lee YK, et al. Genome sequence of "Candidatus Aquiluna" sp. strain IMCC13023, a marine member of the Actinobacteria isolated from an Arctic fjord. J Bacteriol. 2012;194:3550-1.

13. Santoro AE, Dupont CL, Richter RA, Craig MT, Carini P, McIlvin MR, et al. Genomic and proteomic characterization of "Candidatus Nitrosopelagicus brevis": an ammonia-oxidizing archaeon from the open ocean. Proc Natl Acad Sci USA. 2015;112:1173-8.

14. Salcher MM, Neuenschwander SM, Posch T, Pernthaler J. The ecology of pelagic freshwater methylotrophs assessed by a highresolution monitoring and isolation campaign. ISME J. 2015;9:2442-53.

15. Giovannoni SJ, Hayakawa DH, Tripp HJ, Stingl U, Givan SA, Cho JC, et al. The small genome of an abundant coastal ocean methylotroph. Environ Microbiol. 2008;10:1771-82.

16. Jimenez-Infante F, Ngugi DK, Vinu M, Alam I, Kamau AA, Blom $\mathrm{J}$, et al. Comprehensive genomic analyses of the OM43 clade, including a novel species from the Red Sea, indicate ecotype differentiation among marine methylotrophs. Appl Environ Microbiol. 2016;82:1215-26.

17. Chistoserdova L. Modularity of methylotrophy, revisited. Environ Microbiol. 2011;13:2603-22.

18. Chistoserdova L. Methylotrophs in natural habitats: current insights through metagenomics. Appl Microbiol Biotechnol. 2015;99:5763-79.

19. Kalyuzhnaya MG, Bowerman S, Lara JC, Lidstrom ME, Chistoserdova L. Methylotenera mobilis gen. nov., sp. nov., an obligately methylamine-utilizing bacterium within the family Methylophilaceae. Int J Syst Evol Microbiol. 2006;56:2819-23.

20. Govorukhina NI, Trotsenko YA. Methylovorus, a new genus of restricted facultatively methylotrophic bacteria. Int J Syst Bacteriol. 1991;41:158-62.

21. Yordy JR, Weaver TL. Methylobacillus: a new genus of obligately methylotrophic bacteria. Int $\mathrm{J}$ Syst Bacteriol. 1977;27:247-55.

22. Jenkins O, Byrom D, Jones D. Methylophilus - a new genus of methanol-utilizing bacteria. Int J Syst Bacteriol. 1987;37:446-8.

23. Huggett M, Hayakawa D, Rappe M. Genome sequence of strain HIMB624, a cultured representative from the OM43 clade of marine Betaproteobacteria. Stand Genom Sci. 2012;6:11-20.

24. Newton RJ, Jones SE, Eiler A, McMahon KD, Bertilsson S. A guide to the natural history of freshwater lake bacteria. Microbiol Mol Biol Rev. 2011;75:14-49.

25. Woodhouse JN, Kinsela AS, Collins RN, Bowling LC, Honeyman GL, Holliday JK, et al. Microbial communities reflect temporal 
changes in cyanobacterial composition in a shallow ephemeral freshwater lake. ISME J. 2016;10:1337-51.

26. Li J, Zhang J, Liu L, Fan Y, Li L, Yang Y, et al. Annual periodicity in planktonic bacterial and archaeal community composition of eutrophic Lake Taihu. Sci Rep. 2015;5:15488.

27. Ramachandran A, Walsh DA. Investigation of XoxF methanol dehydrogenases reveals new methylotrophic bacteria in pelagic marine and freshwater ecosystems. FEMS Microbiol Ecol. 2015;91:fiv105.

28. Morris RM, Longnecker K, Giovannoni SJ. Pirellula and OM43 are among the dominant lineages identified in an Oregon coast diatom bloom. Environ Microbiol. 2006;8:1361-70.

29. Sekar R, Fuchs BM, Amann R, Pernthaler J. Flow sorting of marine bacterioplankton after fluorescence in situ hybridization. Appl Environ Microbiol. 2004;70:6210-9.

30. Salcher MM, Šimek K. Isolation and cultivation of planktonic freshwater microbes is essential for a comprehensive understanding of their ecology. Aquat Micro Ecol. 2016;77:183-96.

31. Zotina T, Köster O, Jüttner F. Photoheterotrophy and lightdependent uptake of organic and organic nitrogenous compounds by Planktothrix rubescens under low irradiance. Freshw Biol. 2003;48:1859-72.

32. Bolger AM, Lohse M, Usadel B. Trimmomatic: a flexible trimmer for Illumina sequence data. Bioinformatics. 2014;30:2114-20.

33. Bankevich A, Nurk S, Antipov D, Gurevich AA, Dvorkin M, Kulikov AS, et al. SPAdes: a new genome assembly algorithm and its applications to single-cell sequencing. J Comput Biol. 2012;19:455-77.

34. Seemann T. Prokka: rapid prokaryotic genome annotation. Bioinformatics. 2014;30:2068-9.

35. Tatusov RL, Natale DA, Garkavtsev IV, Tatusova TA, Shankavaram UT, Rao BS, et al. The COG database: new developments in phylogenetic classification of proteins from complete genomes. Nucleic Acid Res. 2001;29:22-28.

36. Haft DH, Loftus BJ, Richardson DL, Yang F, Eisen JA, Paulsen Ian T, et al. TIGRFAMs: a protein family resource for the functional identification of proteins. Nucleic Acid Res. 2001;29:41-43.

37. Kanehisa M, Sato Y, Morishima K. BlastKOALA and GhostKOALA: KEGG tools for functional characterization of genome and metagenome sequences. J Mol Biol. 2016;428:726-31.

38. Caspi R, Billington R, Fulcher CA, Keseler IM, Kothari A, Krummenacker M, et al. The MetaCyc database of metabolic pathways and enzymes. Nucleic Acid Res. 2017;46:D633-9.

39. Chistoserdova L, Lapidus A, Han C, Goodwin L, Saunders L, Brettin T, et al. Genome of Methylobacillus flagellatus, molecular basis for obligate methylotrophy, and polyphyletic origin of methylotrophy. J Bacteriol. 2007;189:4020-7.

40. Lapidus A, Clum A, LaButti K, Kaluzhnaya MG, Lim S, Beck DAC, et al. Genomes of three methylotrophs from a single niche reveal the genetic and metabolic divergence of the Methylophilaceae. J Bacteriol. 2011;193:3757-64.

41. Vuilleumier S, Chistoserdova L, Lee M-C, Bringel F, Lajus A, Zhou Y, et al. Methylobacterium Genome Sequences: A Reference Blueprint to Investigate Microbial Metabolism of C1 Compounds from Natural and Industrial Sources. PLOS ONE. 2009;4:e5584.

42. Good N, Lamb A, Beck D, Martinez-Gomez N, Kalyuzhnaya M. C1-Pathways in Methyloversatilis universalis FAM5: Genome Wide Gene Expression and Mutagenesis Studies. Microorganisms. 2015;3:175.

43. Kalyuzhnaya MG, Hristova KR, Lidstrom ME, Chistoserdova L. Characterization of a novel methanol dehydrogenase in representatives of Burkholderiales: implications for environmental detection of methylotrophy and evidence for convergent evolution. J Bacteriol. 2008;190:3817-23.
44. Brautaset T, Jakobsen ØM, Flickinger MC, Valla S, Ellingsen TE. Plasmid-dependent methylotrophy in thermotolerant Bacillus methanolicus. J Bacteriol. 2004;186:1229-38.

45. Vorholt JA, Kalyuzhnaya MG, Hagemeier CH, Lidstrom ME, Chistoserdova L. MtdC, a novel class of methylene tetrahydromethanopterin dehydrogenases. J Bacteriol. 2005; 187:6069-74.

46. Ward N, Larsen Ø, Sakwa J, Bruseth L, Khouri H, Durkin AS, et al. Genomic insights into Methanotrophy: the complete genome sequence of Methylococcus capsulatus (Bath). PLOS Biol. 2004;2:e303.

47. Hou S, Makarova KS, Saw JH, Senin P, Ly BV, Zhou Z, et al. Complete genome sequence of the extremely acidophilic methanotroph isolate V4, Methylacidiphilum infernorum, a representative of the bacterial phylum Verrucomicrobia. Biol Direct. 2008;3:26.

48. Wu ML, Wessels HJCT, Pol A, Op den Camp HJM, Jetten MSM, van Niftrik L, et al. XoxF-type methanol dehydrogenase from the anaerobic methanotroph "Candidatus Methylomirabilis oxyfera". Appl Environ Microbiol. 2015;81:1442-51.

49. Sun J, Steindler L, Thrash JC, Halsey KH, Smith DP, Carter AE, et al. One carbon metabolism in SAR11 pelagic marine bacteria. PLOS ONE. 2011;6:e23973.

50. Denef VJ, Mueller RS, Chiang E, Liebig JR, Vanderploeg HA. Chloroflexi CL500-11 populations that predominate deep-lake hypolimnion bacterioplankton rely on nitrogen-rich dissolved organic matter metabolism and $\mathrm{C} 1$ compound oxidation. Appl Environ Microbiol. 2016;82:1423-32.

51. Li W, Godzik A. Cd-hit: a fast program for clustering and comparing large sets of protein or nucleotide sequences. Bioinformatics. 2006;22:1658-9.

52. Edgar RC. MUSCLE: multiple sequence alignment with high accuracy and high throughput. Nucleic Acid Res. 2004;32: 1792-7.

53. Eddy SR. Accelerated Profile HMM Searches. PLOS Comput Biol. 2011;7:e1002195.

54. Altschul SF, Madden TL, Schäffer AA, Zhang J, Zhang Z, Miller W, et al. Gapped BLAST and PSI-BLAST: a new generation of protein database search programs. Nucleic Acid Res. 1997;25:3389-402.

55. Goris J, Konstantinidis KT, Klappenbach JA, Coenye T, Vandamme P, Tiedje JM. DNA-DNA hybridization values and their relationship to whole-genome sequence similarities. Int $\mathbf{J}$ Syst Evol Microbiol. 2007;57:81-91.

56. Rodriguez-R LM, Konstantinidis KT. Bypassing cultivation to identify bacterial species. ASM Microbe Mag. 2014;9:111-8.

57. Price MN, Dehal PS, Arkin AP. FastTree 2-approximately maximum-likelihood trees for large alignments. PLOS ONE. 2010;5:e9490.

58. Lassmann T, Sonnhammer EL. Kalign—an accurate and fast multiple sequence alignment algorithm. BMC Bioinforma. 2005;6:298.

59. Stamatakis A. RAxML version 8: a tool for phylogenetic analysis and post-analysis of large phylogenies. Bioinformatics. 2014;30:1312-3.

60. Katoh K, Standley DM. MAFFT multiple sequence alignment software version 7: improvements in performance and usability. Mol Biol Evol. 2013;30:772-80.

61. Konstantinidis KT, Rossello-Mora R, Amann R. Uncultivated microbes in need of their own taxonomy. ISME J. 2017;11: 2399-406.

62. Parks DH, Chuvochina M, Waite DW, Rinke C, Skarshewski A, Chaumeil P-A, et al. A standardized bacterial taxonomy based on genome phylogeny substantially revises the tree of life. Nat Biotechnol. 2018;36:996-1004. 
63. Salcher MM, Pernthaler J, Frater N, Posch T. Vertical and longitudinal distribution patterns of differnt bacterioplankton populations in a canyon-shaped, deep prealpine lake. Limnol Oceanogr. 2011;56:2027-39.

64. Bock C, Salcher M, Jensen M, Pandey RV, Boenigk J. Synchrony of eukaryotic and prokaryotic planktonic communities in three seasonally sampled Austrian lakes. Front Microbiol. 2018;9:1290.

65. Linz AM, Crary BC, Shade A, Owens S, Gilbert JA, Knight R, et al. Bacterial community composition and dynamics spanning five years in freshwater bog lakes. mSphere. 2017;2:pii: e00296-17.

66. Okazaki Y, Nakano S-i. Vertical partitioning of freshwater bacterioplankton community in a deep mesotrophic lake with a fully oxygenated hypolimnion (Lake Biwa, Japan). Environ Microbiol Rep. 2016;8:780-8.

67. Cabello-Yeves PJ, Zemskaya TI, Rosselli R, Coutinho FH, Zakharenko AS, Blinov VV, et al. Genomes of novel microbial lineages assembled from the sub-ice waters of Lake Baikal. Appl Environ Microbiol. 2018;84:e02132-02117.

68. Bendall ML, Stevens SLR, Chan L-K, Malfatti S, Schwientek P, Tremblay $\mathrm{J}$, et al. Genome-wide selective sweeps and genespecific sweeps in natural bacterial populations. ISME J. 2016;10: 1589-601.

69. Beck DAC, McTaggart TL, Setboonsarng U, Vorobev A, Kalyuzhnaya MG, Ivanova N, et al. The expanded diversity of Methylophilaceae from Lake Washington through cultivation and genomic sequencing of novel ecotypes. PLoS ONE. 2014;9: e102458.

70. Hendriks J, Oubrie A, Castresana J, Urbani A, Gemeinhardt S, Saraste M. Nitric oxide reductases in bacteria. Biochim Biophys Acta. 2000;1459:266-73.

71. Mustakhimov I, Kalyuzhnaya MG, Lidstrom ME, Chistoserdova L. Insights into denitrification in Methylotenera mobilis from denitrification pathway and methanol metabolism mutants. J Bacteriol. 2013;195:2207-11.

72. Kalyuzhnaya MG, Lapidus A, Ivanova N, Copeland AC, McHardy AC, Szeto E, et al. High-resolution metagenomics targets specific functional types in complex microbial communities. Nat Biotechnol. 2008;26:1029-34.

73. Kitzinger K, Padilla CC, Marchant HK, Hach PF, Herbold CW, Kidane AT, et al. Cyanate and urea are substrates for nitrification by Thaumarchaeota in the marine environment. Nat Microbiol. 2019;4:234-43.
74. Wetzel R: Limnology. Lake and river ecosystems. 3rd ed. San Diego: Elsevier Academic Press; 2001.

75. Halsey KH, Carter AE, Giovannoni SJ. Synergistic metabolism of a broad range of $\mathrm{C}_{1}$ compounds in the marine methylotrophic bacterium HTCC2181. Environ Microbiol. 2011;14:630-40.

76. Chistoserdova L. Methylotrophy in a lake: from metagenomics to single-organism physiology. Appl Environ Microbiol. 2011;77:4705-11.

77. Pinhassi J, DeLong EF, Béjà O, González JM, Pedrós-Alió C. Marine bacterial and archaeal ion-pumping rhodopsins: Genetic diversity, physiology, and ecology. Microbiol Mol Biol Rev. 2016;80:929-54.

78. Steindler L, Schwalbach MS, Smith DP, Chan F, Giovannoni SJ. Energy starved Candidatus Pelagibacter ubique substitutes lightmediated ATP production for endogenous carbon respiration. PLOS ONE. 2011;6:e19725.

79. Luecke H, Schobert B, Stagno J, Imasheva ES, Wang JM, Balashov SP, et al. Crystallographic structure of xanthorhodopsin, the light-driven proton pump with a dual chromophore. Proc Natl Acad Sci USA. 2008;105:16561-5.

80. Imasheva ES, Balashov SP, Choi AR, Jung K-H, Lanyi JK Reconstitution of gloeobacter violaceus rhodopsin with a lightharvesting carotenoid antenna. Biochemistry. 2009;48:10948-55.

81. Rusch DB, Halpern AL, Sutton G, Heidelberg KB, Williamson S, Yooseph S, et al. The Sorcerer II Global Ocean Sampling Expedition: Northwest Atlantic through Eastern Tropical Pacific. PLoS Biol. 2007;5:e77.

82. Béjà $\mathrm{O}$, Aravind L, Koonin EV, Suzuki MT, Hadd A, Nguyen LP, et al. Bacterial Rhodopsin: evidence for a new type of phototrophy in the sea. Science. 2000;289:1902-6.

83. Logares R, Bråte J, Bertilsson S, Clasen JL, Shalchian-Tabrizi K, Rengefors $\mathrm{K}$. Infrequent marine-freshwater transitions in the microbial world. Trends Microbiol. 2009;17:414-22.

84. Walsh DA, Lafontaine J, Grossart H-P. On the eco-evolutionary relationships of fresh and salt water bacteria and the role of gene transfer in their adaptation. In: Gophna U editor. Lateral gene transfer in evolution. New York: Springer; 2013. p. 55-77.

85. Zhang H, Yoshizawa S, Sun Y, Huang Y, Chu X, González JM, et al. Repeated evolutionary transitions of flavobacteria from marine to non-marine habitats. Environ Microbiol. 2019;0: 648-66.

86. Roberts MF. Organic compatible solutes of halotolerant and halophilic microorganisms. Saline Syst. 2005;1:5. 